\title{
RBF-FD BASED DYNAMIC THERMAL RATING OF OVERHEAD POWER LINES
}

\author{
GREGOR KOSEC \& JURE SLAK \\ Parallel and Distributed Systems Laboratory, "Jožef Stefan” Institute, Slovenia
}

\begin{abstract}
The essential limiting factor of the power transmission line transfer capabilities is the maximal allowed temperature of the conductor that should not be exceeded to avoid excessive sags. A commonly used conservative approach is to limit transfer capability to the worst case scenarios, i.e. hot, sunny, windless days. Of course, system operators strive to raise the limit with more sophisticated models that take into account actual weather conditions or even weather forecasts. As a consequence, there has been substantial research done on Dynamic Thermal Rating (DTR) models in the last few decades. Based on accumulated knowledge the leading standards in the field published guidelines for thermal rating for operative use. However, the proposed models rely only on empirical relations for determination of the temperature gradient on the surface of the conductor that dictates the heat flux due to the advection. This heat flux is the most intense cooling mechanism in play, and also the most complex to model. In this paper, we extend the discussion about advective cooling with a direct simulation of temperature and velocity fields near the conductor with the focus on the natural convection regime. The introduced model considers joule heat generation and heat transport within the power line and its vicinity, fluid flow driven by buoyancy force, solar heating, and radiation. The solution procedure uses RBF-FD numerical method combined with Poisson disk sampling nodal positioning algorithm. The results of the simulation are presented in terms of temperature and velocity magnitude contour plots, convergence analyses, and comparison of convective heat losses of simulated results to IEC, IEEE and CIGRE standards.
\end{abstract}

Keywords: overhead power line, DTR, meshless, natural convection.

\section{INTRODUCTION}

The increasing demands for electrical power pressure the transmission system operators to improve transmission capabilities. As a result, some existing lines might become overloaded, especially in summer time, causing bottlenecks that can lead to blackouts [1].

The essential limiting factor of the power transmission line transfer capabilities is the maximal allowed temperature of the conductor, e.g. $80^{\circ} \mathrm{C}$ in the Slovenian power system, which should not be exceeded to avoid excessive sags. A commonly used conservative approach is to limit transfer capability to the worst case scenarios, i.e. hot, sunny, windless days. Of course, system operators strive to raise the limit with more sophisticated models that take into account actual weather conditions or even weather forecasts.

As a consequence, a substantial research has been done on Dynamic Thermal Rating (DTR) models in the last few decades [2]-[4] that consider heat generation within the conductor and heat exchange with surroundings due to various mechanisms, namely radiation, solar heating, rain impinging and the most important as well as the most complex to model: convective heat transport. The most widely accepted models are collected in leading CIGRE [5], IEEE [6] and IEC [7] standards that offer comprehensive overview of research done in the field.

The CIGRE, IEEE and IEC model the convective cooling by means of empirical relations that relate Grashof, Prandtl and Nusselt numbers, i.e. state relation between the power of cooling in dependence on the material properties and the temperature difference between the cylinder and the ambient. The most accepted is relation provided by Morgan [8], however 


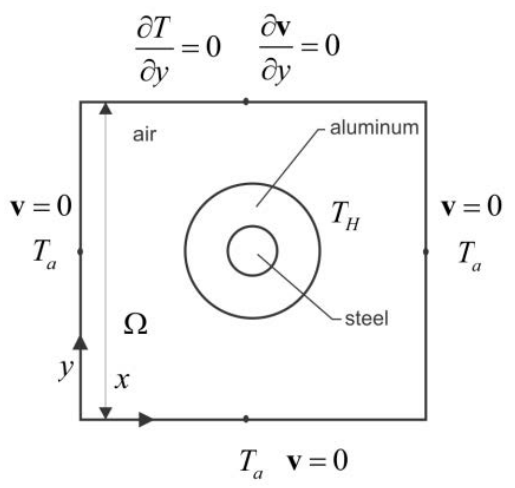

Figure 1: Scheme of the problem.

there are also other similar relations as Churchill and Bernstein relation [9], McAdams relation [10], Zukauskas and Žiugžda relation [11], etc.

In this paper we present a physical model of heat transfer from power line that does not rely solely on the data provided by measurements but tries to predict the thermal state from thermo-physical properties of air, aluminum, and steel and geometry of the power line. The introduced model is solved numerically using a local meshless RBF-FD [12] based on weighted least squares approximation [13], [14]. In this paper only natural convection regime is discussed.

The rest of the paper is organized as follows, in Section 2, Physical model, we discuss the main phenomena in the model, in Section 3 the solution procedure RBF-FD method for simulation of the physical model is presented, and in Section 4, Results, we discuss the computed results.

\section{PHYSICAL MODEL}

First, a physical model for thermal rating of overhead power line is discussed. The domain of the problem is a cross-section of a power line that is further separated into a steel core and aluminum conductor, and surrounding air (Fig. 1). Within the steel core only heat conduction takes place

$$
c_{p}^{s t} \rho^{s t} \frac{\partial T^{s t}}{\partial t}=\lambda^{s t} \nabla^{2} T^{s t}
$$

while heat generation due to Joule losses is also present in the aluminum conductor

$$
c_{p}^{a l} \rho^{a l} \frac{\partial T^{a l}}{\partial t}=\lambda^{a l} \nabla^{2} T^{a l}+q_{J}
$$

where indices st, al denote steel and aluminum domain, $c_{p}$ specific heat capacity, $\lambda$ thermal conductivity, $\rho$ density, and $q_{j}$ heat source due to the Joule losses, which is modeled as

$$
q_{j}=\frac{I^{2} R(T)}{S^{a l}}
$$

with temperature dependent conductivity $R(T)$ introduced as [5] 


$$
R(T)=R_{20}\left(1+\alpha_{20}\left(T-20^{\circ} \mathrm{C}\right)\right),
$$

where $R_{20}$ stands for resistivity at $20^{\circ} \mathrm{C}, \alpha_{20}=4.5 \mathrm{e}-3^{\circ} \mathrm{C}^{-1}$ for the thermal resistance coefficient, and $S^{a l}$ for conductor area.

The heat transport in surrounding air is driven primarily by convection and therefore momentum transport has to be considered, which is modelled with the Navier-Stokes equation and mass continuity further coupled with the heat transfer through the Boussinesq approximation

$$
\begin{gathered}
\nabla \cdot \mathbf{v}=0 \\
\rho \frac{\partial \mathbf{v}}{\partial t}+\rho \nabla \cdot(\mathbf{v v})=-\nabla P+\nabla \cdot(\mu \nabla \mathbf{v})+\mathbf{b} \\
\rho \frac{\partial\left(c_{p} T\right)}{\partial t}+\rho \nabla \cdot\left(c_{p} T \mathbf{v}\right)=\nabla \cdot(\lambda \nabla T), \\
\mathbf{b}=\rho\left[1-\beta_{T}\left(T-T_{\text {ref }}\right)\right] \mathbf{g}
\end{gathered}
$$

with $\mathbf{v}(u, v), P, T, \lambda, c_{p}, \mathbf{g}, \rho, \beta_{T}, T_{\text {ref }}, \mu$ and $\mathbf{b}$ standing for velocity, pressure, temperature, thermal conductivity, specific heat, gravitational acceleration, air density, the coefficient of thermal expansion, reference temperature for Boussinesq approximation, viscosity and body force, respectively. Thermal conductivity and viscosity of air are further modelled as [5]

$$
\begin{aligned}
& \lambda=2.368 \cdot 10^{-2}+7.23 \cdot 10^{-5} T-2.763 \cdot 10^{-8} T^{2}, \\
& \mu=\left(17.239+4.635 \cdot 10^{-2} T-2.03 \cdot 10^{-5} T^{2}\right) \cdot 10^{-6} .
\end{aligned}
$$

On boundaries between steel core, aluminum conductor and surrounding air, conservation has to hold yielding following boundary conditions

$$
\begin{gathered}
T^{a l}\left(r_{1}\right)=T^{s t}\left(r_{1}\right), \\
\left.\lambda^{a l} \frac{\partial T^{a l}}{\partial \mathbf{n}}\right|_{r_{1}}=\left.\lambda^{s t} \frac{\partial T^{s t}}{\partial \mathbf{n}}\right|_{r_{1}}, \\
T^{a l}\left(r_{2}\right)=T^{a}\left(r_{2}\right), \\
\left.\lambda^{a l} \frac{\partial T^{a l}}{\partial \mathbf{n}}\right|_{r_{2}}-\left.\lambda \frac{\partial T}{\partial \mathbf{n}}\right|_{r_{2}}=q_{r},
\end{gathered}
$$

where $r_{1}$ in $r_{2}$ denote steel core and line radius, $\mathbf{n}$ outside unit normal vector to the domain boundary, and $q_{r}$ heat sink due to the radiation, modelled as

$$
q_{r}=-\sigma_{B} \varepsilon_{s}\left(T_{s}^{4}-T_{a}^{4}\right),
$$


where $\sigma_{B}=5,67\left[\mathrm{~W} / \mathrm{m}^{2} \mathrm{~K}^{4}\right]$ and $\varepsilon_{s}$ stand for the Stefan-Boltzmann constant and emissivity, respectively. $T_{s}$ and $T_{a}$ stand for skin temperature $\left(T_{s}=T^{a l}\left(r_{2}\right)\right)$ and ambient temperature.

At the top boundary the symmetry is assumed

$$
\begin{aligned}
& \left.\frac{\partial \mathbf{v}}{\partial y}\right|_{\text {top }}=0, \\
& \left.\frac{\partial T}{\partial y}\right|_{\text {top }}=0 .
\end{aligned}
$$

Other boundaries are assumed to be far enough that air is still at ambient temperature.

\section{SOLUTION PROCEDURE}

The solution procedure is divided in two time loops, namely the air and the power line loop. The reason for this lies in extremely different dynamics of involved phenomena. For illustration, if skin temperature of line suddenly increases for $40^{\circ}$, the system (5)-(8) will reach steady state in few seconds. On the other hand, if electric current through the conductor suddenly increases for $800 \mathrm{~A}$, the power line will reach thermal equilibrium only in a few hours. This can be quickly seen from values of thermo-physical properties introduced in next section. Besides, the system (1)-(4) can be solved implicitly with a relatively big time step, i.e. in order of $10 \mathrm{~s}$, while non-linearities of the system (5)-(8) requires much finer temporal stepping, i.e. in order of $1 \mathrm{~ms}$. Therefore, first system (1)-(4) is solved implicitly with time step $\Delta t_{\text {line }}$ using values of air temperature from previous time step, followed by internal iteration, where system (5)-(8) is solved explicitly with $\Delta t_{\text {air }}$.

Each internal time step begins with computing the intermediate velocity ( $\mathbf{v}^{\text {iter }}$ ) from eqn (6) without pressure term. Since the intermediate velocity does not satisfy eqn (5), a Poisson pressure correction equation

$$
\nabla^{2} p^{\text {corr }}=\frac{\rho}{\Delta t_{\text {air }}} \nabla \cdot \mathbf{v}^{\text {iter }}
$$

is solved with

$$
\frac{\Delta t}{\rho} \frac{\partial p^{c o r r}}{\partial n}=\mathbf{n}\left(\mathbf{v}^{\text {iter }}-\mathbf{v}^{B C}\right)
$$

boundary condition. The pressure Poisson equation is, at given boundary conditions, defined only up to a constant and to avoid instabilities a unique solution is enforced with an additional condition, also referred to as a regularization

$$
\int_{\Omega} p d \Omega=0
$$

Once the pressure correction is known, a velocity is corrected accordingly

$$
\mathbf{v}^{\text {corr }}=-\frac{\Delta t}{\rho} \nabla P^{\text {corr }}
$$


Finally, the eqn (7) is solved, again with Euler's method. The internal iteration takes place until steady state is achieved.

Spatial discretization needed to compute involved partial differential operators is based on a local approximation of a considered field over the overlapping local support domains, i.e. in each node an approximation over a small local sub-set of neighboring $n$ nodes among all nodes $N$

$$
\hat{u}(\mathbf{p})=\sum_{i}^{m} \alpha_{i} b_{i}(\mathbf{p})=\mathbf{b}(\mathbf{p})^{\mathrm{T}} \boldsymbol{\alpha}
$$

with $m, \boldsymbol{\alpha}, \mathbf{b}, \mathbf{p}\left(p_{x}, p_{y}\right)$ standing for the number of basis functions, approximation coefficients, basis functions and the position vector, respectively, is used. In this paper we use a higher number of support nodes than the number of basis functions, i.e. $n>m$, and therefore weighted least squares (WLS) approximation is used to solve the over-determined problem, i.e. a norm

$$
r^{2}=\sum_{j}^{n} W\left(\mathbf{p}_{j}\right)\left(u\left(\mathbf{p}_{j}\right)-\hat{u}\left(\mathbf{p}_{j}\right)\right)^{2}=(\mathbf{B} \boldsymbol{\alpha}-\mathbf{u})^{\mathrm{T}} \mathbf{W}(\mathbf{B} \boldsymbol{\alpha}-\mathbf{u}),
$$

is minimized, where $\mathbf{W}$ is a diagonal matrix with elements $W_{j j}=W\left(\mathbf{p}_{j}\right)$ with

$$
W(\mathbf{p})=\exp \left(-\left(\frac{\left\|\mathbf{p}_{0}-\mathbf{p}\right\|}{\sigma p_{\min }}\right)^{2}\right)
$$

where $\sigma$ stands for weight parameter, $\mathbf{p}_{0}$ for the center of support domain and $p_{\min }$ for the distance to the first support domain node. The solution can be written in matrix form as

$$
\boldsymbol{\alpha}=\left(\mathbf{W}^{0.5} \mathbf{B}\right)^{+} \mathbf{W}^{0.5} \mathbf{u}
$$

where $\left(\mathbf{W}^{0.5} \mathbf{B}\right)^{+}$stand for a Moore-Penrose pseudo inverse. By explicit expression of $\boldsymbol{\alpha}$ into an equation

$$
\hat{u}(\mathbf{p})=\mathbf{b}(\mathbf{p})^{\mathrm{T}}\left(\mathbf{W}^{0.5}(\mathbf{p}) \mathbf{B}\right)^{+} \mathbf{W}^{0.5}(\mathbf{p}) \mathbf{u}=\chi(\mathbf{p}) \mathbf{u},
$$

is obtained, where $\chi$ stand for the shape function. Now, we can apply partial differential operator $(L)$, on the trial function,

$$
L \hat{u}(\mathbf{p})=L \chi(\mathbf{p}) \mathbf{u},
$$

and obtain an approximation for desired operator at point $\mathrm{p}$. In described model, we deal with Navier-Stokes and Heat equations and therefore only shape functions for Laplace operator and first derivatives are needed, which are pre-computed and stored.

\section{RESULTS}

The natural convection from 490-AL1/64-ST1A power line is numerically investigated in this section. This power line with radius $1.33 \mathrm{~cm}$ is made of material with following properties $\rho_{a l}=2703 \mathrm{~kg} / \mathrm{m}^{3}, \rho_{s t}=7780 \mathrm{~kg} / \mathrm{m}^{3}, \quad c_{p}^{a l}=897 \mathrm{~J} /(\mathrm{kgK}) \quad c_{p}^{a l}=481 \mathrm{~J} /(\mathrm{kgK})$. The 
thermal conductivity of the power line is estimated to $\lambda^{a l}=\lambda^{a l}=2 \mathrm{~W} / \mathrm{mK}$ as proposed in [5], [15]. The air is modelled with $\rho=1.29 \mathrm{~kg} / \mathrm{m}^{3}, c_{p}=1005 \mathrm{~J} / \mathrm{kgK}$ and $\beta=0.00367$. In the numerical simulation the 490-AL1/64-ST1A is positioned in the centre of in $10 \mathrm{x} 10 \mathrm{~cm}$ square domain.

We start analysis with illustration of phenomena by means of contour plots of temperature field and velocity magnitude near power line (Fig. 2).

First set of more quantitative analysis is focused on internal iteration, i.e. on computation of thermo-fluid problem near power line at constant skin temperature. In Fig. 3 temperature profiles for different $\Delta T=T_{s}-T_{a}$ are presented, where we can see that the boundary layer is in order of a few centimeters, and as expected it narrows with increasing difference between skin and ambient temperature. From Fig. 4 it can be concluded that the presented RBF-FD solution is convergent and that, as assumed in solution procedure section, the steady state is achieved within few seconds for all reasonable cases.
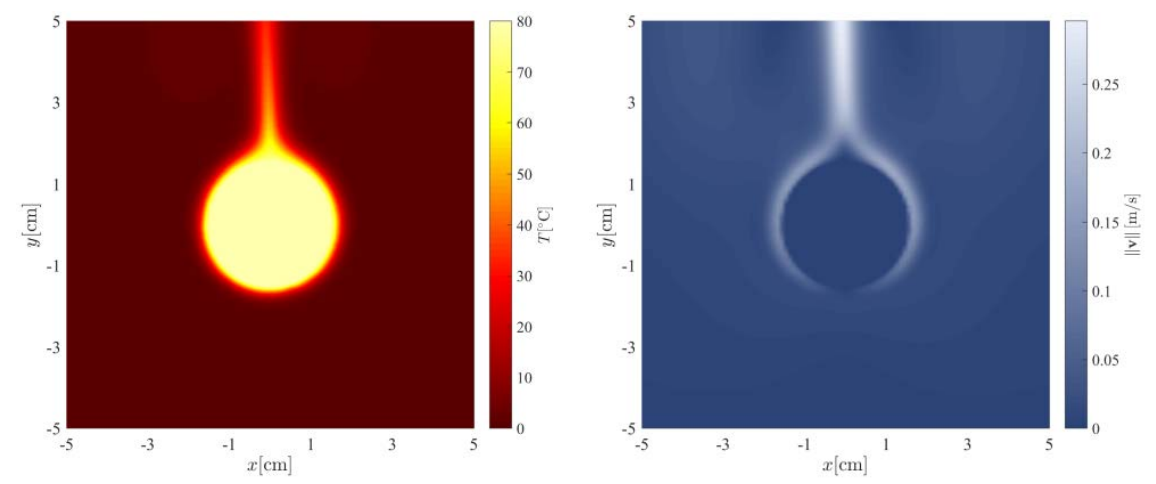

Figure 2: Temperature (left) and velocity magnitude (right) contour plots for cooling of overhead power line.

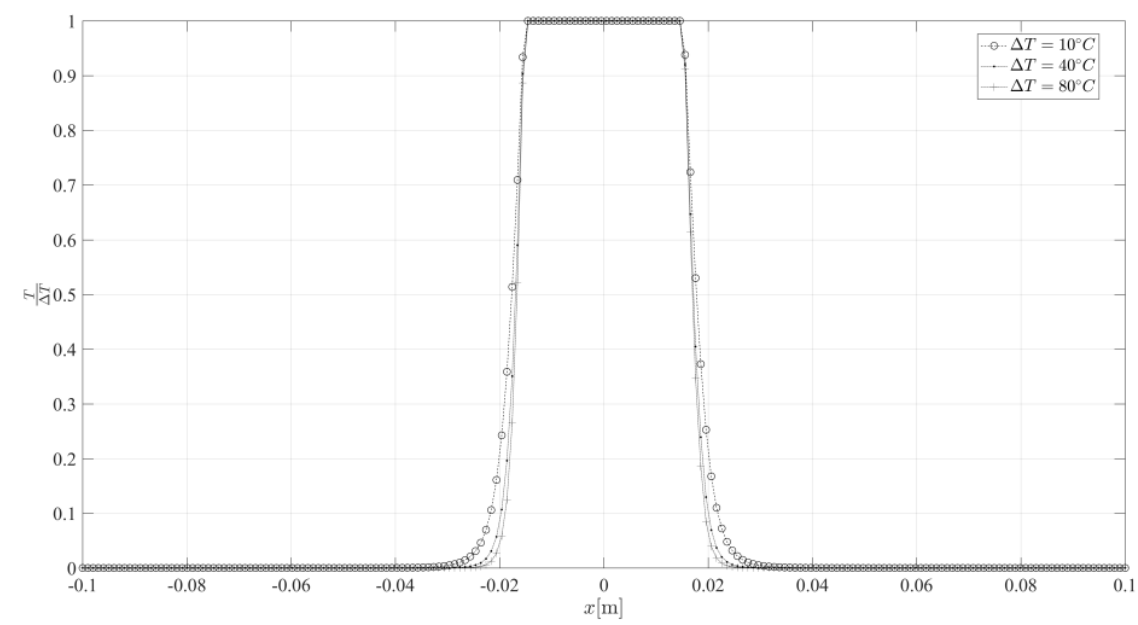

Figure 3: Temperature profile $T(x, y=0)$ at different $\Delta T=T_{s}-T_{a}$. 

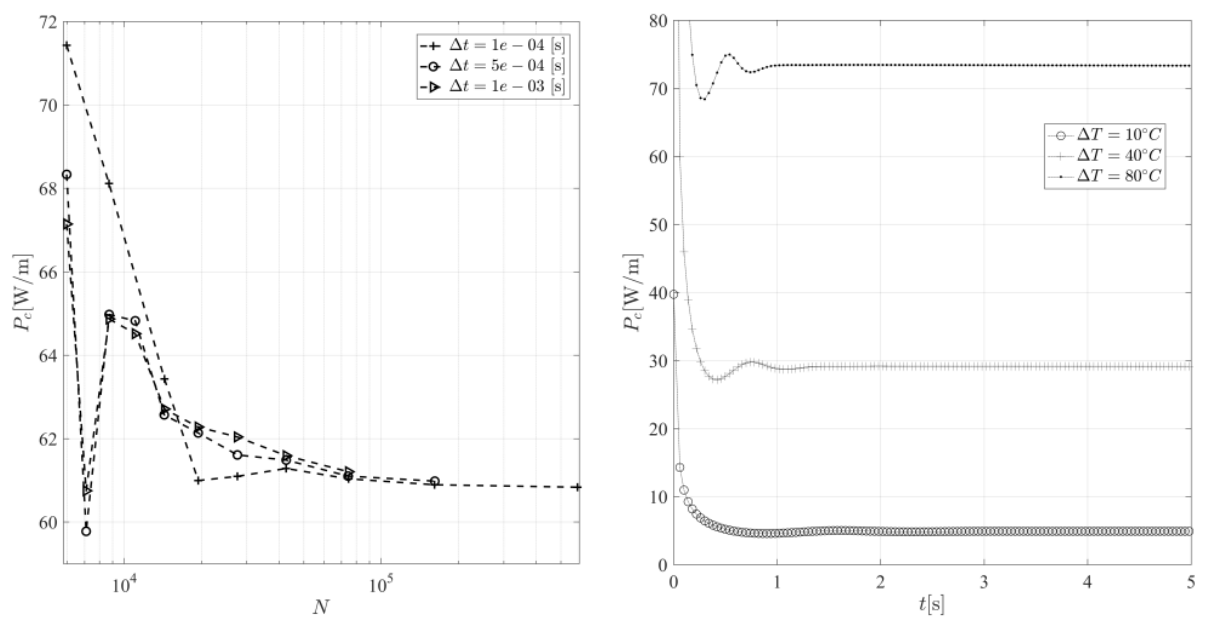

Figure 4: Convergence plot (left) and time development for different $\Delta T=T_{s}-T_{a}$.

In the left plot of Fig. 5 a comparison of RBF-FD simulation, CIGRE, IEEE, and IEC models in terms of convective cooling as a function of $\Delta T=T_{a}-T_{S}$ is presented. It can be clearly seen that IEC completely fails at prediction, while CIGRE, IEEE and RBF-FD simulation agree relatively well. In the right plot of Fig. 5 a full simulation is presented. Two different runs are presented in one figure. In each run electric current two times suddenly increases and then suddenly drops to zero. This way we cover four heating simulations and two cooling simulations. A comparison of RBF-FD simulation CIGRE model gives relatively good agreement.
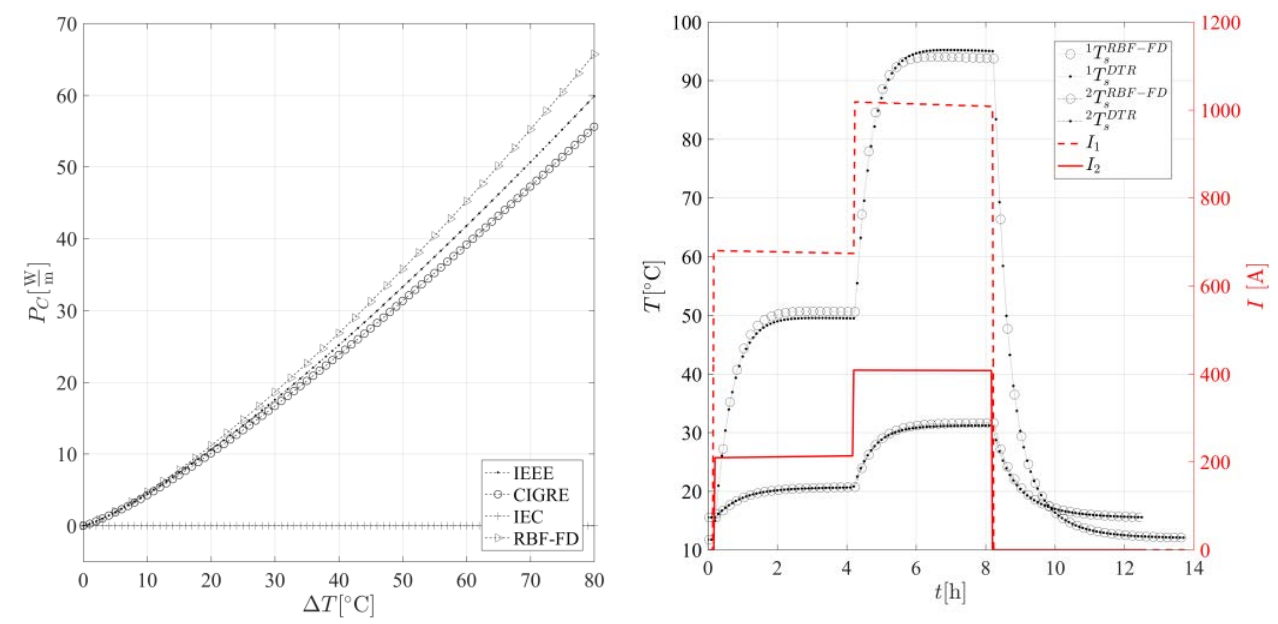

Figure 5: Power of convective cooling with respect to the difference between the skin temperature and ambient temperature $\left(\Delta T=T_{a}-T_{S}\right)$ (left) and skin temperature development (right). 


\section{CONCLUSIONS}

This paper introduces relatively simple physical model describing the natural convection from overhead power line that takes into account heat transport and generation within the power line, radiation on the line skin, and thermo-fluid transport in the surrounding air. The presented physical model is numerically solved by RBF-FD meshless numerical method and it is demonstrated that simulated cooling by natural convection agrees well with the results provided by CIGRE and IEEE standards. In future work we want to further analyze the phenomena by simulating natural convection from different power lines and to include also forced convection in the analysis.

\section{ACKNOWLEDGEMENTS}

The authors would like to acknowledge the financial support of ELES, Ltd., Electricity Transmission System Operator, and Slovenian Research Agency (ARRS) in the framework of the research core funding No. P2-0095.

\section{REFERENCES}

[1] Berizzi, A., The Italian 2003 blackout. IEEE Power Engineering Society General Meeting, 2004.

[2] Kosec, G., Maksić, M. \& Djurica, V., Dynamic thermal rating of power lines: Model and measurements in rainy conditions. International Journal of Electrical Power \& Energy Systems, 91, pp. 222-229, 2017.

[3] Pytlak, P., Musilek, P., Lozowski, E. \& Toth, J., Modelling precipitation cooling of overhead conductors. Electric Power Systems Research, 81, pp. 2147-2154, 2011.

[4] Morgan, V.T., The thermal rating of overhead-line conductors. Electric Power Systems Research, 5, pp. 119-139, 1981.

[5] CIGRE, Guide for Thermal Rating Calculations of Overhead Lines, 2014.

[6] IEEE Standard for Calculating the Current-Temperature Relationship of Bare Overhead Conductors, 2014.

[7] International Electrotechnical Technical Committee 20, Overhead Electrical Conductors, 2016.

[8] Morgan, V., The overall convective heat transfer from smooth circular cylinders. Advances in Heat Transfer, 1, pp. 199-264, 1975.

[9] Churchill, S.W. \& Bernstein, M., A correlating equation for forced convection from gases and liquids to a circular cylinder in crossflow. J. Heat Transfer, 99(2), pp. 300306, 1977.

[10] McAdams, W.H., Heat Transmission, McGraw-Hill: New York, 1959.

[11] Zukauskas, A. \& Žiugžda, J., Heat Transfer of a Cylinder in Crossflow, Hemisphere Publications, 1985.

[12] Bollig, E.F., Flyer, N. \& Erlebacher, G., Solution to PDEs using radial basis function finite-differences (RBF-FD) on multiple GPUs. Journal of Computational Physics, 231, pp. 7133-7151, 2012.

[13] Slak, J. \& Kosec, G., Refined meshless local strong form solution of Cauchy-Navier equation on an irregular domain. Engineering Analysis with Boundary Elements, in press. DOI: https://doi.org/10.1016/j.enganabound.2018.01.001.

[14] Kosec, G., A local numerical solution of a fluid-flow problem on an irregular domain. Advances in Engineering Software, 120, pp. 36-44, 2018.

[15] Morgan, V.T., The radial temperature distribution and effective radial thermal conductivity in bare solid and stranded conductors. IEEE Transactions on Power Delivery 5, pp. 1443-1452, 1990. 